\section{ICMC 2007 Keynote Address}

Fifty Years of Computer Music:

Ideas of the Past Speak to a Future Immersed in Rich Detail

\section{by Fohn Chowening}

CCRMA

Department of Music

Stanford University

\section{ABSTRACT}

Two early uses of the computer to synthesize and process sound-additive and FM synthesis - led to new thoughts about sound spectra, tuning and pitch. Detached from their traditional association with the timbre of acoustic instruments, spectra become structured and associated with pitch in ways that are unique to the medium of computer music

With 50 years of rapid technological evolution and increasing knowledge has come an explosion of detail, which can become tractable through imaginative use of the inherent attributes of the medium itself and through visualizations that can make implicit detail explicit.

\section{INTRODUGTION}

In 1957, just fifty years ago, Max Mathews introduced a wholly new means of making music. An engineer/scientist at Bell Telephone Laboratories (BTL), Max (with the support of John Pierce, who was director of research) created out of numbers and code the first music to be produced by a digital computer. It is usually the case that a fascination with some aspect of a discipline outside of one's own will quickly conclude with an experiment without elaboration. But in Max's case, it was the beginning of a profoundly deep and consequential adventure. It was an adventure that he modestly invited us all to join through his elegantly conceived programs, engendering tendrils that found their way into far-flung disciplines that continue to grow without end fifty years later.

From the very beginning, Max's use of the computer for making music was expansive. Synthesis, signal processing, analysis, algorithmiccomposition,psychoacousticsall were within his scope, and all were expressed and described in great detail in his famous article [4] and the succession of programs MUSIC I-V. ${ }^{1}$

It is in the nature of the computer medium that detail be elevated at times to the forefront of our thinking. Unlike preceding music technologies, both acoustic and analogue, computers require us to manage detail to accomplish even the most basic steps. It is in the detail that we find control of the sonic, theoretical and creative forms. And it is through paying attention to detai that we reveal our scientific/engineering insights or our artistic expression-our own voice.

The first examples of computer-generated music produced by Max Mathews and John Pierce at BTL were rich in ideas, including algorithmic composition, novel tuning, matching tuning systems to complementary spectra, imaginative and compelling graphics and visualizations and, soon afterward, controllers [3]. It is fortunate that these two scientists/engineers - who cultivated a nexus between science and art, and who invited composers and artists like Varèse and Cage to their laboratories to share the possibilities that they sawwere willing to place these nascent musical studies in the public view, confident in the intellectual content of their ideas. Some of their ideas remain as compelling now a they were then and should be "re-viewed" given the enriched domains of application at this 50-year mark.

\section{BREAKTHROUGHS}

The richness of the ideas in these early examples was not matched by the quality of the sounds with which they were expressed. Little was known about some important aspects of perception and the acoustics of musical instruments. Max invited two important composers to work at BTL, both of whom made important contributions in this area in addition to creating compositions. They were James Tenney and Jean-Claude Risset.

Preceding Max's famous article by a few months was an article by Tenney that described in exquisite detail the program that Max had created [8]. Tenney had been invited by John Pierce and Max to work at BTL beginning in 1961. He had studied with the visionary Lejaren Hiller at the University of Illinois, so he came prepared in matters of programming and stochastic processesin composition. During his three years at BTL, he made several important contributions. $\mathrm{He}$ created compositions using this new medium, and he wrote in great detail about how he had constructed his compositions and what he had learned from Max. Because he was a composer, Tenney's description of Max's MUSIC IV was from a musical viewpoint, and it remains an exemplar of clarity and completeness. ${ }^{2}$

But, important to the points being presented in this paper, Tenney came upon a musicdriven question in his compositions using MUSIC IV for which there was no answer. So, with Max's guidance, he did a study regarding the perception of attack times [8] 
The italicization is to draw attention to two points: first, the fact that from the outset psychoacoustics had been seen by Max as one of the crucial disciplines in the advancement of computer music ${ }^{3}$, and second, that musicians have a particular sensitivity to the details of auditory perception.

\section{Risset Uncovers the Microstructure}

There is no doubt that the most important breakthrough in the early days of computer music occurred when Jean-Claude Risset and Max Mathews began detailed computer studies in the analysis, synthesis and perception of acoustic instrument tones, which culminated in Risset's $A n$ Introductory Catalogue of Computer Synthesized Sounds [3]. With this work, the medium of computer music reached a level beyond Max's correct but abstract assertion that computers (coupled with loudspeakers) can produce any perceivable sound. The capability of simulating natural-sounding tones presupposes an understanding of the perceptual relevance of the physical stimuli, only some of which have been "selected" as meaningful by the auditory system.

Risset began his research with a study of trumpet tones [7]. The brass family had eluded attempts at simulation based upon extant acoustic descriptions. In a detailed analysis, Risset discovered that the "signature" of the trumpet tone is the correlation of input force (pressure) and spectral bandwidth. The evolution of the harmonic amplitudes during the attack portion of the tone is rapid, complicated, but with a direction: as the overall intensity of the tone increases, the greater is the relative contribution of the higher harmonics. Risset had made an important discovery relating the auditory system's perception of "naturalness" to selected physical stimuli. This discovery figured prominently in the advancement of FM synthesis.

\section{FM Synthesis - 40 Years}

It was forty years ago that this author "stumbled" upon FM synthesis [1]. The actual date is not known. Not having a scientific or engineering background, I did not have the habit of keeping dated lab notes, but I did keep notes. There is a record of my having visited BTL on December 18, 1967, when I showed the data that I used in my first trials to Max, Risset and Pierre Ruiz and played them the examples. It was a month or two before, almost certainly late at night, while experimenting with extreme vibrato frequencies and depths that I realized "there is more here than at first meets the ear."

Its discovery was not a purposeful searchthat is, stemming from a realization, from looking at the equation, that there might be some interesting experiments to try Rather, it was altogether a discovery of the "ear."

One must remember that while the theoretical potential for the production of rich dynamic sounds with the computer was great, the knowledge required for realizing this potential was meager. Risset's catalogue was in progress and was little known outside of BTL. Furthermore, the cost in computer time was enormous, limiting the complexity of synthesis algorithms. Deep into the details of digital reverberation at the time, I was keenly aware of this issue. My "ear" was continually scanning for any sound having internal dynamism, coupled oscillators, random vibrato, etc. That I found it within such a computationally efficient algorithm was certainly partly chance, but then I was also certainly prepared for that chance.

The first experiments were each only of a few seconds' duration, because of the ten of minutes' computation time on a timeshared system. But they do show that from the outset, all of the essential features that would eventually be developed and used in musical contexts were noted:

- both harmonic and inharmonic spectra could be produced

- a change in frequency deviation $(\Delta \mathrm{f})$ produced a change in bandwidth of the spectrum
- the spectrum is conserved through the pitch space with a constant ratio of FM frequencies

As it turned out, these parameters of FM synthesis have a remarkable perceptual relevance.

As mentioned above, Risset's study of trumpet tones had a major influence on my own development of FM synthesis. I first heard about this study on the aforementioned visit to BTL in 1967, during which I showed my first experiments in FM synthesis. Risset explained his analysis and re-synthesis of trumpet tones and played some examples. It was not until 1970, however, that I fully appreciated the importance of his discoveries about trumpet tones.

While working on the FM synthesis of percussive sounds, I noted that in nearly all tones of this class, the amplitude envelope and the envelope controlling the modulation index were very similar if not identical. I also noted that there was as strong a correlation of the perception of "strike force" to the modulation index as there was to intensity. I considered other classes of tones where this might be the case, and I remembered Risset's explanation of the "signature" of trumpet tones, some three years previous. With only a few attempts I was able to create credible brass-like tones by simply coupling a single 


\section{array}

ICMC 2007 Keynote Address function to the amplitude and modulation index envelopes with appropriate scaling. I realized that this correlation of force or effort (strike force, breath and bow pressure velocity, etc.) to the bandwidth and/or high-frequency emphasis of partials can be generalized to all natural sound and that the parameters of FM synthesis provided a straightforward implementation of this important correlation. ${ }^{4}$

Then began a rapid development of FM synthesis ${ }^{5}$, and the eventual licensing of the technology by Stanford University to Yamaha. The rest is history.

\section{STRUCTURED SPECTRA PITCH SPACE}

There are two ways in which additive synthesis and FM synthesis have been used that merit emphasis, because they touch upon issues that are important beyond any particular means of synthesis. John Pierce and Max foresaw one way in the early years: the creation of a non-traditiona scale that has a structural link to timbre, where the frequency ratios from the scale are used in the construction of the tone's spectra. $^{6}$ Risset, however, used synthesis in a manner not foreseen - a manner imaginative and evocative.

Constructing Spectra in the Pitch Space

The final example in Risset's catalogue stands as a striking advance in computer music, although it is little recognized and little exploited. It is the first instance where pitch is used to express timbre in the same functional manner that pitch expresses melody and harmony - melodyharmony-timbre all within the pitch space. Pitch is composed sequentially as line and simultaneously as harmony, for which there are rich functional theories, but composing timbre as a collection of partials drawn from the pitch space cannot be achieved with acoustic instruments and falls squarely in the domain of computer music.

The sound potential of any instrument is vast, but limited - the partials that make up an instrument's tone can only be partly modified by performance techniques and devices such as mutes. A clarinet and a violin can play the same pitch at the same loudness for the same duration, but they cannot be made to have the same spectrum through time - the frequency and intensity of an instrument's partials are locked within boundaries defined by its and the performer's physical properties.

Risset realized in his timbre studies that by summing numbers of sinusoids (pure tones) where each sinusoid can have its own independent control over intensity and frequency through time, he had unlocked timbre from any physical constraints. He could create tones that cannot exist in the natural world, complex timbres where the partials themselves are a part of the pitch space. He composed a short pitch sequence that is heard first sequentially in time (melody), then simultaneously in time (harmony), and then again simultaneously with exactly the same pitches but now as partials associated with a single sound source, as shown in Figure 1. [2]
Because all of the partials die away in a similar manner, they fuse and are heard as timbre rather than harmony. The timbre is similar to that of a gong, but a gong whose spectrum is imprinted with pitch nformation, giving the sound an extranatural structural link to the preceding Risset's was an altogether new conception, uniquely possible with computers, and beautifully framed in several of his compositions, first in Mutations (1969).

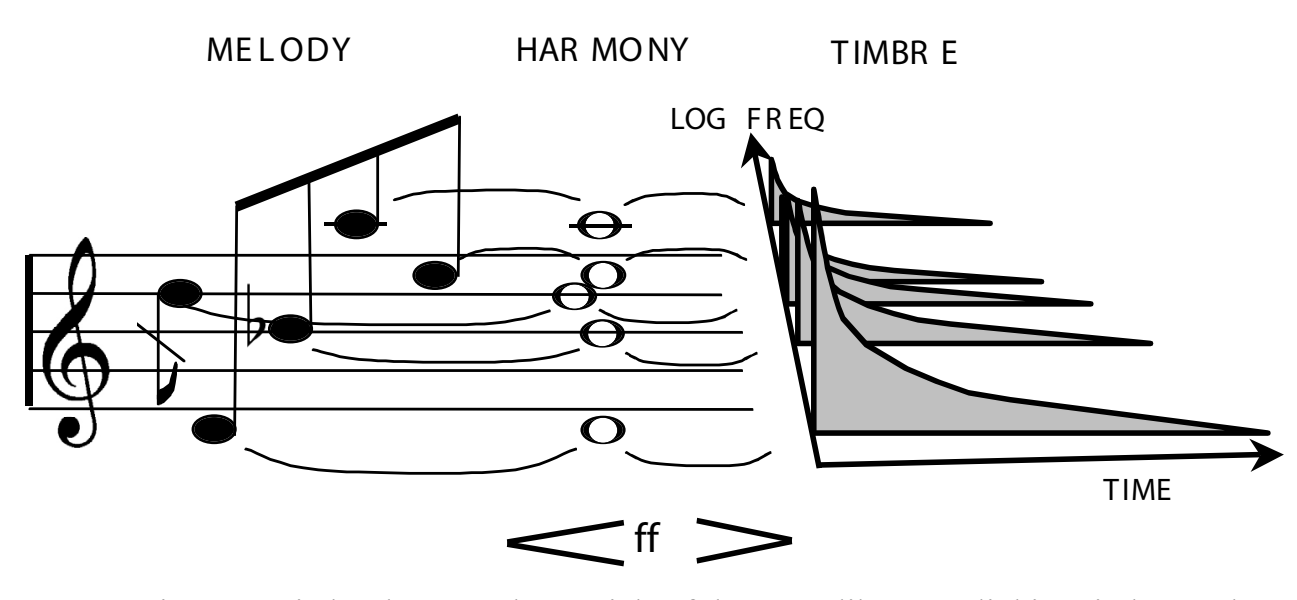

Figure 1. Pitches become the partials of the gong-like tone, linking timbre to the pitch space in a manner uniquely possible with computers. From Jean-Claude Risset's Mutations (1969). 
Constructing Spectra and the Pitch Space

John Pierce and Max saw early on that using the computer for both control and synthesis could unlock tuning systems from physical constraints, just as Risset had unlocked timbre. Max composed a piece, The Second Law, that is entirely made up of noise and entirely free of common

understandings of pitch, yet it expresses pitch. In his Eight-Tone Canon (1966) [3], Pierce divided the octave into eight equal steps. The even-numbered steps (equal to the multiples of three in a twelve-step division) and odd-numbered steps each form a diminished seventh chord. But what is interesting about this short piece is that Pierce used tones composed of sums of sinusoids

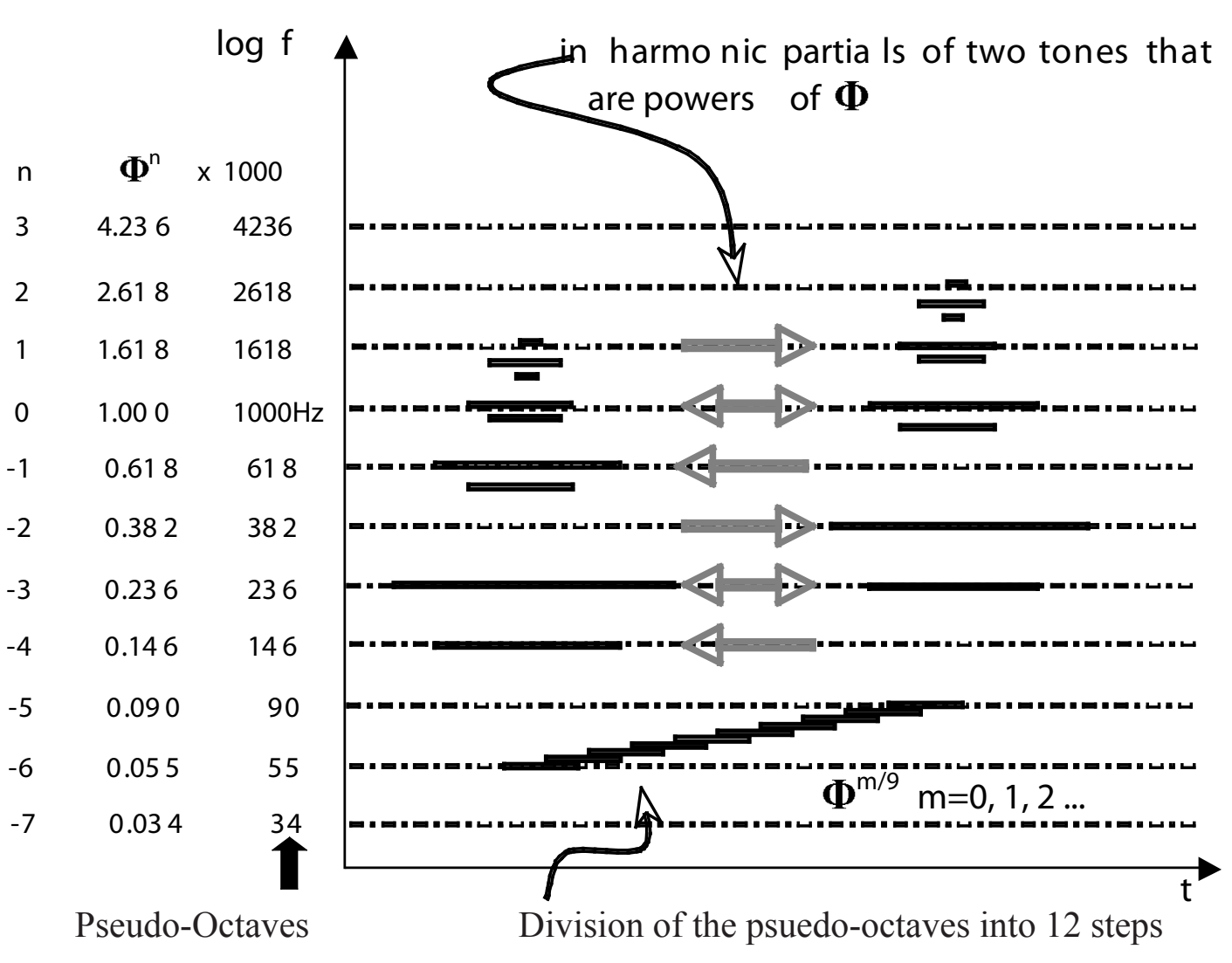

Figure 2. The pitch space in both Stria and Voices is based upon pseudo-octaves that are powers of the Golden Ratio $\phi$, which are further divided into 9 steps. The spectra of tones generated by FM c:m ratios that are also powers of $\phi$ contain partials that are powers of $\phi$, here shown at the interval of a pseudo-octave. that progress from octave to half-octave to quarter octave with each iteration of the canon. Except for the octave, the spectra are inharmonic, but they are composed of frequencies that are common to the pitch space!

\section{Stria (1977)}

While this example is not rich in the sonic sense, it is a compelling and powerful idea that I found especially evocative because of my interest in spectra composed of ordered inharmonic partials that could be simply produced by FM synthesis. One class of such spectra that I found particularly interesting is based upon carrier-to-modulator frequency ratios $\left(\mathrm{f}_{\mathrm{c}} / \mathrm{f}_{\mathrm{m}}\right)$ derived from the Golden Ratio or $\phi \approx 1.618$. Remembering Pierce's canon, I conceived a composition in the mid 1970s that is based upon spectra structured in a way that is complementary to the division of the pitch space. The traditional octave is replaced by a pseudo-octave based upon powers of the Golden Ratio $\left(\phi^{\mathrm{n}}\right)$ rather than powers of 2 , and the spectra are produced by values of $f / f$ that are also powers of $\phi$ as can be seen in Figure 2.

After several years thinking about its theoretical underpinnings, I realized Stria in the months from July to October 1977. It was first presented on October 13 at the Centre Pompidou as part of IRCAM' concert series "La Voix des voies," produced by Luciano Berio.
The Fall 2007 issue of the Computer Music fournal (31:3) contains a suite of articles about Stria that fully explain the circumstances of its composition, the several versions and analyses. ${ }^{7}$ The Winter issue of CMJ (31:4) includes a DVD with all of the original materials that were used by the authors in their articles, the original quad sound files, a reconstruction in quad sound files and a visualization of Stria from the Princeton Sound Lab.)

\section{Voices v. $3(2007)^{8}$}

Vices for soprano and interactive computer uses the same division of the pitch space and structured spectra as Stria. Again, all of the sounds are produced by FM synthesis and all of the spectra are generated from ratios based on $\phi$, as noted above (except for a few instances of voice-like tones that use integer ratios). The formal structure of Voices is altogether different, however, and requires a larger set of differentiated sounds than did Stria.

The important and initial question was how well a soprano, both as a performer and as a "sound," would fit into this "artificial" pitch/spectral space where, first, the scale is unfamiliar to the performer and not related to any of the common modes or tunings, and second, the partials of sung vowel tones are harmonic and do not 
share the same spectral distribution. Can one mix a sonic artifact totally dependent upon the computer for its existence with a natural-perhaps the most naturalmusical sound, the singing voice?

The music performance problem would seem to be a major hurdle for the soprano While a single scale step in the 9-step/ pseudo-octave division only differs from the traditional semi-tone by 7 cents, the maximum difference in the progression is nearly a quartertone and there are 13 steps in the interval closest to a true octave, as shown in Table 1.

\begin{tabular}{|c|c|c|c|c|c|}
\hline n & $\begin{array}{l}\text { Voices } \\
\text { Scale }\end{array}$ & Pitch & Cents & $\begin{array}{c}\text { Common } \\
\text { Scale }\end{array}$ & $\mathrm{n}$ \\
\hline 0 & .000 & $\mathrm{a}$ & & 000 & 0 \\
\hline & .055 & a\#(-) & -7 & .059 & \\
\hline$\frac{2}{2}$ & .113 & $\mathrm{~b}(-)$ & -14 & .122 & 2 \\
\hline $\begin{array}{l}3 \\
4\end{array}$ & $\begin{array}{l}.114 \\
.238\end{array}$ & $\begin{array}{l}c(-) \\
c \#(-)\end{array}$ & $\begin{array}{l}-22 \\
-29\end{array}$ & $\begin{array}{r}.189 \\
260\end{array}$ & $\frac{3}{4}$ \\
\hline 5 & .306 & $\mathrm{~d}(-)$ & -37 & .335 & 5 \\
\hline 6 & .378 & $\mathrm{~d} \#(-)$ & -44 & .414 & 6 \\
\hline $\begin{array}{l}7 \\
8 \\
\end{array}$ & .454 & $\mathrm{~d} \#(+)$ & 48 & .414 & 6 \\
\hline 9 & $\begin{array}{l}.534 \\
618\end{array}$ & $f(+)$ & $\begin{array}{l}41 \\
34\end{array}$ & .580 & 8 \\
\hline 0 & .707 & $\mathrm{f}(+)$ & 26 & .682 & 9 \\
\hline & .801 & $\mathrm{~g}(+)$ & 19 & .782 & 10 \\
\hline 2 & 1.900 & $\mathrm{g \#}(+)$ & 11 & 1.888 & 0 \\
\hline 13 & 2.004 & $a(+)^{\prime}$ & 4 & 2.000 & 2 \\
\hline
\end{tabular}

Table 1. A comparison of the scale used in Voices, $\phi^{n / 9}$, with the common tempered scale, $2^{\text {n/2 }}$, showing the closest scale degree and the difference in cents. The $6^{\text {th }}$ step of the common scale repeats to maintain the proximate Voices step and show that it is the 13th step of the Voices scale that is closest to a true octave.
It is my good fortune to have had a soprano ${ }^{9}$ at hand with whom I could work during the initial stages of the composition. My hypothesis was that singing in this unusual scale is possible if the structured inharmonic spectra of the accompanying tones are infused with complementary pitch information, since most good performers tune to context. I included in the program (written in Max/MSP) the option for the singer to give herself a cue tone for the current target pitch or the following target pitch. In fact, the option is rarely used, since the singer seems to easily tune to the partials of the structured spectra - to the partials of the structured
context, as hypothesized.

$6^{\text {th }}$

12

.

The other part of the initial question was this: how well would the soprano, having partials in the harmonic series, fit within a context composed of dense (albeit structured) inharmonic partials? The somewhat surprising answer is that the performer and the listener are unaware of any spectral mismatch. Moreover, one senses an overall pitch coherence that is more like a soprano singing with an ensemble of acoustic instruments with harmonic spectra than with idiophones having dense inharmonic spectra (such as gongs and bells). There are several possible reasons that there is no overall perception of "out-of-tuneness" or psychoacoustic dissonance.

While the spectra of low tones in Voices are often dense, they are selectively dense, with partials of a single tone spaced at intervals from large to small (in log frequency), as is the case with the harmonic series. In addition, the spectra are composed such that the energy is concentrated around the low order partials (small modulation index). Therefore, whether or not low order partials fall within a critical band is dependent upon the interval of two tones within the pitch space, as is the case with partials in the harmonic series.

Another reason is that the concentration of harmonic energy in typical soprano tones is limited to the low order harmonics, especially the fundamental, which reduces the incidence of strong partials interacting within critical bands.

Finally, there is a third, somewhat speculative reason why there is little psychoacoustic dissonance. Critical band theory is based upon perceptual experiments using stimuli having few variables and highly stable partials, quite unlike the sounds of the natural listening experience. We know that the auditory system responds to partials in a different way when mediating temporal factors such as amplitude envelopes or synchronous micro-modulation (e.g. random or quasiperiodic vibrato) are present.

This internal dynamism brings into play an additional perceptual theory based upon grouping and common fate from the Gestalt laws of perceptual organization. It is a higher-level mechanism (probably not in the cochlea) that causes partials to fuse or cohere where individual partials are difficult or impossible to distinguish, and they become identifiable as a source, known or unknown, and segregable within a collection of sources.

It seems that these temporal features, which are intrinsic to our "out of lab" perceptual experience, may reduce the importance of the interaction of individual partials relative to critical bands, because the interaction becomes transitory and no longer stable. 
Dynamic partials not only animate the sounds (harmonic or inharmonic) of which they are a part, but they contribute to the surface allure of the larger sound context, leading the ear through time in a complex of detailed multi-dimensional spaces of timbre, location, loudness andmost importantly regarding dissonancepitch. The pitch space is loosened from its abstract skeletal form by the internal dynamic detail and accommodates the sound of the soprano, whose fluid expression derives from its own internal dynamism.

During the thirty years since composing Stria, I have often wondered whether the integrated spectral tuning and pitch tuning worked because of the particular attributes of the work itself, the manner in which the work slowly unfolds from sparse to dense spectra having enormous acoustic mass, having no other spectral forms than those rooted in the Golden Ratio. Is it a pitch/spectrum construct that is unique to the piece?

My experience with Voices suggests that it is not. It could be that these early ideas - Risset's structured spectra, Max's and Pierce's joining of odd tunings with complementary spectra and Max's evolving pitch space in his melodic metamorphoses - can be generally exploited with synthesized sound, with physical models where the "physical" is infinitely malleable, or even sampled sounds, especially with the availability of new stable high-Q filters [6]. A medium is defined by its distinctive attributes, and these ideas are certainly unique to music made with computers.

\section{IMMERSED IN DETAIL MANAGING IT ALL}

From the micro-structural detail involved in understanding, creating and processing the sound itself, to the understanding of machine-generated or -processed music for which the description (the equivalent to a score) may lie in the detail of program code (which, even if familiar, is not a springboard to formal visualization), to the vast amount of relevant theoretical, empirical and poetic detail that is available to us through the web, technology provides us with ever more options and consequent detail - we are immersed.

But the evolving technology also provides us with increasing power and new options for managing the detail - links that become vast virtual libraries, rich in content constructed by experts - and new ways to make accessible the complexity of detail when it is transformed into synchronous visual/auditory representations.

\section{Knowledge Webs}

I have long been impressed with Julius
Smith's Global fOS Index and its everevolving context on the CCRMA server. Rich in detail and having great scope, it is a resource to which I refer often. For example, whenever asked the difference between frequency and phase modulation, I refer the inquirer to this index. This is a detail that I need to know about, but not all the time and certainly not in the same way that Julius Smith knows about it. In these matters, Julius Smith is an expert whereas I am not, and the answer is better found in a rich but neutral context. It is a detail that may have quite different meaning for the inquirer, inviting investigation of related topics that could lead to unanticipated results.

The Max/MSP-Pd concept of "help files" is a localized but extremely effective web providing interactive sound examples in addition to text descriptions. But this web and the Global FOS Index should be linked, and this is precisely what Julius Smith and his colleagues plan to do. His concept of "knowledge webs" includes:

- "backwards learning"(start reading about what you want to know, and click as needed)

- on-line, demand-driven, self-paced education

- complete "ladders" from any research topic down to the advanced high-school level
What an idea! This growing community, of which we are a part, can strive to enhance and tighten the links that connect us and to enable those from whom we have yet to hear. $^{10}$

\section{Visualization}

Since the early days, Max and his colleagues have effectively used graphic representation of processes both as a means of composing - signal bandwidth as a function of time or functional control of melodic transformation - and as a means of simplifying complex processes. Indeed, the very form of diagrammatic representation of unit generators and instruments that Max used in his Music I-V programs presaged the object-oriented programs that are now so widely used. It was a representation that made computer music accessible to those of us who were not expert programmers.

Once-difficult concepts, loaded with relevant detail, can now be easily explained in a very short time, thanks to sound-synchronous animation. For example, with two or three animated slides, the relationship between Bessel function coefficients and the amplitudes of the sideband components (partials) when the modulation index is changing as a function of time can be demonstrated and understood to the required level of usefulness in minutes as opposed to 
hours. All such demonstrations should be integrated into the "Knowledge Web" and become available to our growing community of users.

Making visible the relevant detail of the music that we compose and listen to is an exciting frontier. The Princeton Sound $\mathrm{Lab}^{11}$ provided me with a modification of 'sndpeek,' their real-time, multi-mode sound visualization program with which one can display in real-time any prerecorded music. My request was for a special concert visualization of Stria. The modification extends the 3-D spectral display to include the future as well as the past, so that the waterfall display has an illuminated slice in the middle that is the "now line." This representation, somewhere between signal and score, provides the listener with a view of what $i$ to come, thus revealing details of Stria, e.g. the complex beating and the ambiguity between frequency and pitch, that would be nearly impossible to show in any other visualization and that are only implicitin the score-generating code and the score itself While there is only twice the information when compared to a typical 3-D waterfall projection, the perceived detail seems much more, as one is able to track details in both the future and the past. There are a number of further refinements planned that will bring this representation even closer to how we perceive. Doug Keislar, who has a long-standing interest in music representation and who has suggested many refinements, points out that this kind of inter-modal reinforcement can be especially appropriate to electroacoustic music, for which there is typically no score. ${ }^{13}$

\section{GONCLUSION}

Extending and better understanding the ideas of structured spectra and complementary tuning spaces could lead to dynamic contextual tuning where the spectrum and pitch spaces are malleable through time, but always coherent.

Composers can reveal the background detail of their work, as poetic as it is technical, through the use of graphics and new modes of visualization of compositional structures. With the sound and/or score it becomes a complete record - the meta-composition - and it al becomes part of our "Knowledge Web."

\section{NOTES}

1. For a complete account of Max Mathews' work and publications, see http://www. ina.fr/produits/publications/collections/ collec_11.fr.html.

2. His early interest and important contributions notwithstanding, Jim Tenney did not continue in computer music, but rather became a distinguished teacher, performer and composer of acoustic music.
He died on August 24, 2006.

3. Max wrote in 1963 "At present, the range of computer music is limited principally by cost and by our knowledge of psychoacoustics [4]."

4. The ease with which spectral change could be coupled to effort (key velocity) is one of the reasons for the YAMAHA DX7's remarkable success.

5. The first real-time FM synthesis was programmed on a DEC PDP-15 computer by Barry Truax in 1973, while studying in Utrecht. At Stanford, Bill Schottstaed developed a particularly powerful form of the algorithm that was used in many compositions for many years.

6. Karlheinz Stockhausen created a similar relationship between pitch and spectrum in his Studie 1 (1953), although with analog technology.

7. "An Analysis of the Compositional Techniques in John Chowning's Stria" by Matteo Meneghini; "The assembling of Stria by John Chowning: A philological investigation" by Laura Zattra; "A Reconstruction of Stria" by Olivier Baudouin; and "Surface Tensions: Dynamics of Stria " by Kevin Dahan. 8. Voices was commissioned by GRM in 2004 and first performed (version 1) by Maureen Chowning at the Maison de Radio, Paris, March 12, 2005.

9. Maureen Chowning, for whom Voices is written, has had experience singing in alternative tunings, e.g. Solemn Songs for Evening by Richard Boulanger, written in the Pierce-Bohlen scale.

10. See http://ccrma.stanford.edu/ realsimple/

11. PhD candidates Ge Wang and Ananya Misra, and Perry Cook, Director.

13. 2003 IEEE Workshop on Applications of Signal Processing to Audio and Acoustics October 19-22, 2003, New Paltz, NY

\section{REFERENGES}

[1] Chowning, J. "The Synthesis of Complex Audio Spectra by Means of Frequency Modulation.” F. Audio Eng. Soc. 21, 71973.

[2] Chowning, J. "Composing the Sound Itself," Portraits Polychrome, Vol. 142, No. 3592, 553-557, 1963.

[3] Goebel, J. (producer). "The Historical CD of Digital Sound Synthesis." Computer Music Currents 13, Schott Wergo, 1995.

[4] Mathews, M. V. "The Digital Computer as a Musical Instrument." Science, Vol. 142, No. 3592, 553-557, 1963.

[5] Mathews, M.V., John R. Pierce, Current Directions in Computer Music Research. Cambridge, MA: MIT Press, 1989.

[6] Mathews, M.V., J. O. Smith, "Methods for synthesizing very high $\mathrm{Q}$ parametrically well behaved two pole filters." In Proceedings of the Stockholm 
Musical Acoustics Conference (SMAC-03), http://www.speech.kth.se/smac03/, Stockholm, Royal Swedish Academy of Music, Aug. 2003. Available online with sound examples at http://ccrma.stanford. edu/ jos/smac03maxjos.

[7] Risset, J.C. "A Computer Study of Trumpet Tones." Fournal of the Acoustical Society of America 38 (1965), p. 912. First presented at the 70th meeting of the Acoustical Society of America, Saint Louis, November, 1965. The study was also included in "Analysis of MusicalInstrument Tones," Physics Today, Vol. 22, No 2, Feb. 1969. With M. V. Mathews as co-author.

[8] Tenney, James C. "SoundGeneration by Means of a Digita Computer." Fournal of Music Theory, Vol.7, No.1, Spring, 1963. (Available http:// links.jstor.org/).
A Selection of Responses to Gregory Taylor's Letter about Gender and Computer Music from the Winter 2006 Issue

The Listening Room (http://dao cim3.net/cgi-bin/wiki.pl?Womens Listening_Room), organized

and curated by Pamela Madsen, demonstrates the wide range of women creating electroacoustic music today (2007). This yearly presentation at California State University-Fullerton is inspiring and could change perceptions about women and the composing of electronic music. The Listening Room is designed for non-stop all-day playback in fine concert halls. The production could be presented anywhere in the world with high quality sound systems and is a great step forward in encouraging women to shape a new musical paradigm. The Listening Room certainly inspired me all over again.

--Pauline Oliveros

At FTM-8 (Feminist Theory \& Music 8 , June 23-26, 2005 in NYC), a letter from Mara Helmuth was distributed to the audience. I find two quotes from that letter to be still very much relevant and important today:

"More often, devaluation of a woman's work is done subtly, by a casual comment implying the insignificance of nonlegitimacy, a joke, or a concert ignored."

and

"We all grow up in a biased culture, and all absorb it in different ways. It is up to us to honestly assess how to change it personally within our lives."

--Paula Matthusen

Computer Music exists at the intersection of engineering, mathematics, and music, all of which have been historically male dominated fields with strongly patriarchal models of pedagogy and achievement. Alarmingly, Anita Borg [1] citesa downward trend in the percentage of CS and CE degrees earned by women. Elizabeth Hinkle-Turner [2], while identifying significant achievements of women in music technology, also notes a decrease in women's participation in the field. It is our job as artists and educators to examine the pedagogical tools and curricula rigorously to ensure that talented voices are not being excluded, and that those who enter the field are encouraged to stay [3]. 